\title{
Characteristics of Stereo Imaging using Non-Identical Cameras for Object Detection
}

\author{
Ahmed M. D. E. Hassanein, Amira H. N. AboElanen, and Salma Ahmed H. Z.
}

\begin{abstract}
The fear from the continuous spreading of the Covid-19 pandemic had put lot of restrictions on the movement of goods around the world. In Egypt, the importing of goods especially electronic products from many countries including China was crucial to the research and educational purposes. The restrictions had stopped the importing of many electronic devices from China including cameras. Object detection and identification were among the hot topics of research in our university which depended mainly on imported cameras. In this paper we tackle the problem of setting up stereo cameras using old non identical cameras to do object detection. The selection of the cameras was not optional since we had to use what we found in our old laptops. OpenCV and Python programming commands were used to set the two cameras to obtain equally clear images as much as possible. A disparity map was then calculated using openCV and its accuracy was then discussed. Accuracy was dependable on the sharpness of the cameras used, Gamma parameter, number of pixels per image and matching algorithm to match the two images obtained using the stereo cameras.
\end{abstract}

Keywords - Disparity, Stereo Camera, OpenCV, Object Detection, Identification.

\section{INTRODUCTION}

Due to the spreading of the Covid-19 pandemic around the world, the importing of goods from foreign countries including China had been severely reduced in Egypt [1]. The problem of object detection had been under research in our university and the use of identical cameras to obtain stereo images of objects was crucial. However, the reduction in, and in some cases halting of, importing goods including cameras from certain countries had left us with no option but to use old cameras from our houses which are not identical. In this paper, we discuss the conditions which we found necessary and crucial when using old non-identical cameras to obtain stereo images which would increase the probability of correct object detection. Being non identical comes from the differences in the type of each camera including focal length, age of the lens of each camera, etc. [2]. This would impose limitations on the detection accuracy of the objects in the obtained images and also the distance calculation from the camera to each detected object [3]. This paper was organized into five sections. In first section, we discussed the types of cameras used and their initial set up. The characteristics of one of the cameras in terms of number of pixels (px) was shown in second section. In the third section, the functions

Submitted on November 20, 2021

Published on December 19, 2021.

Ahmed M. D. E. Hassanein, Systems and Information Department, Engineering Division, National Research Centre (NRC), Dokki, Giza, Egypt.

(e-mail: ahmed.diaa.hassanein ${ }^{@}$ gmail.com). used to setup the stereo cameras were discussed. The numerical results obtained were illustrated in the fourth section. In section five, the conclusions were drawn.

\section{SYSTEM SETUP}

For the calculations done in this paper, we used the pycharm IDE for python programming which was an open source [4]. We downloaded the openCV (cv2) and numpy libraries [5] which proved to be crucial in our calculations.

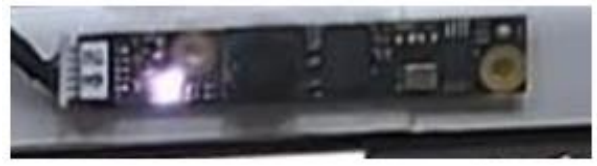

(a)

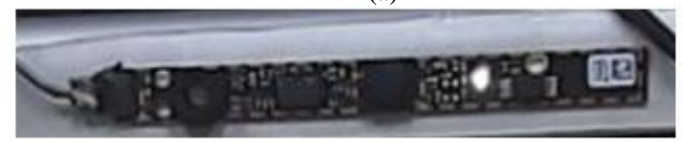

(b)

Fig. 1. An image of the two cameras used attached to a holder. (a): Right Camera. (b) Left Camera.

In this implementation, we used two non-identical cameras which were removed from their respective old laptops and attached them to a holder as shown in Fig. 1. One of them was taken from an Asus Laptop and was defined in its driver as "usb-Chicony Electronics Co. Ltd" as shown in Fig 1a. The other one was taken from an HP laptop and was defined as "usb-Chicony HP High Definition Webcam" by its driver as shown in Fig. 1b. Each camera weighed almost three grams which make them suitable for our hand-held applications. The cameras were taken from old laptops and so no information or user manuals were found for them. After searching the internet, we tried to guess the number of pixels per image which can be obtained from each camera. By trial and error, we programmed both of them to have a resolution of $(640 \mathrm{x} 480) \mathrm{px}$ which was found to be suitable for both cameras. The sharpness of the latter camera was set to a high number (100) as it was the one with the lower number of pixels as we guessed from our internet search. We wanted to increase its sharpness as much as possible to match the sharpness of the other camera. The command in cv2 namelyset(cv2.CAP_PROP_SHARPNESS,100) was used [5]. The focal length was set to autofocus so each camera set its focus value according to the taken image. Next, we studied the characteristics of one of the cameras and see the effect

Amira H. N. AboElanen, MSA University, Egypt.

(e-mail: amira.hassan1@msa.edu.eg).

Salma Ahmed H. Z., MSA University, Egypt.

(e-mail: salma.ahmed13@msa.edu.eg). 
they would have on the performance of our system's object detection.

\section{Characteristics OF Single CAMERA}

In this section, we needed to obtain the values of several parameters manually so as to help us in defining the suitable conditions for the different types of object detections as discussed later. We started by calculating the angle of horizontal view for both cameras. Since we set the resolution to be the same for both cameras and both were set to autofocus then the horizontal angle of view was the same for both. Two images were taken by one of the cameras for two rulers put at right angle at two different distances as shown in Fig. 2.

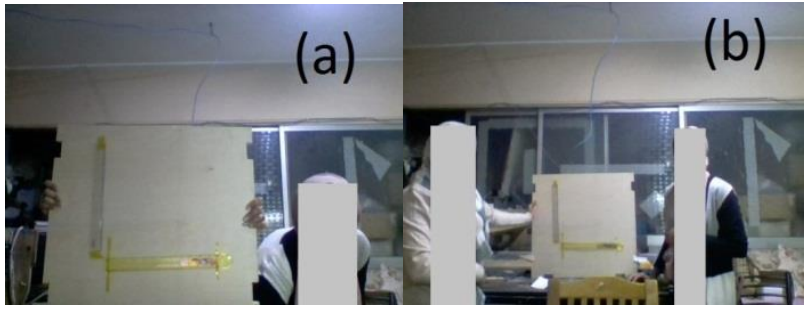

Fig. 2. Images of two perpendicular rulers at two different distances from the camera. (a) Rulers at $1 \mathrm{~m}$ and (b) Rulers at $2 \mathrm{~m}$.

For the image in Fig. 2a, the resolution equation was [6]:

$$
\begin{aligned}
\text { res } 1 & =\frac{\text { total_no_pixels }}{\text { Area_pictures }} \\
& =\frac{(480 \times 640)}{(80 \times 105)}=36.57 \mathrm{px} / \mathrm{cm}^{2}
\end{aligned}
$$

where the "total_no _ pixels" referred to the total number of px in the collected images using the cameras. " Area _ pictures" was the area of the pictured seen in the collected images.

The horizontal angle of view for the camera was [6]:

$$
\begin{aligned}
\phi_{0}=\tan ^{-1}\left(\frac{\text { opposite }}{\text { adjacent }}\right) & = \\
\tan ^{-1}\left(\frac{(1.05 / 2)}{1}\right) & \approx 55^{\circ}
\end{aligned}
$$

where "opposite" was equal to half the horizontal distance covered in the image. "adjacent" was the distance from camera to the pictured seen in the images.

For the image in Fig. 2b, the resolution was [6]:

$$
\begin{aligned}
\text { res2 } & =\frac{\text { total_no_pixels }}{\text { Area_pictures }} \\
& =\frac{(480 \times 640)}{(160 \times 200)} \\
& =9.6 \mathrm{px} / \mathrm{cm}^{2}
\end{aligned}
$$

Then the rate of change of resolution with respect to change of distance from camera to object:

$$
\begin{aligned}
& \frac{d(\text { resolution })}{d z}= \\
& \frac{d(\text { total_no_pixels } / \text { Area_pictures })}{1} \\
& =\text { res } 2-\text { res } 1=-26.97 \mathrm{px} / \mathrm{cm}^{2} / \mathrm{m}
\end{aligned}
$$

where $d$ (resolution) was the change in resolution from equations (1) to equation (3). $d z$ is the difference in distances between res 1 in equation (1) and res 2 in equation (3). Resolution decreased as the distance from camera to object increased and so the ability of our system to correctly detect objects decreased. In ref [7], we found that the average width of a human face was almost $14.76 \mathrm{~cm}$ and the average length was almost $11.7 \mathrm{~cm}$ [7]. For recognition of human face, we needed to have images with $600 \mathrm{px} /$ face [8]. So, the required resolution in this case would be:

$$
\begin{aligned}
\text { res_recogn } & =\frac{\text { total_no_pixels }}{\text { Area_pictures }} \\
& =\frac{600 \mathrm{px} / \text { face }}{(11.7 \times 14.76) / \text { face }} \\
& =3.47 \mathrm{px} / \mathrm{cm}^{2}
\end{aligned}
$$

For identification in good conditions of human faces, we needed to have images having 2000px/face [8]. So, the required resolution in this case would be:

$$
\begin{aligned}
r e s_{-} \text {ident }(\text { good }) & =\frac{\text { total_no_pixels }}{\text { Area_pictures }} \\
& =\frac{2000 \mathrm{px} / \text { face }}{(11.7 \times 14.76) / \text { face }} \\
& =11.58 \mathrm{px} / \mathrm{cm}^{2}
\end{aligned}
$$

For identification in bad conditions of human face, we needed to have images having $8000 \mathrm{px} /$ face [8]. So, the required resolution in this case would be:

$$
\begin{aligned}
\text { res_ident }(\text { bad }) & =\frac{\text { total_no_pixels }}{\text { Area_pictures }} \\
& =\frac{800 \text { px } / \text { face }}{(11.7 \times 14.76) / \text { face }} \\
& =46.32 \mathrm{px} / \mathrm{cm}^{2}
\end{aligned}
$$

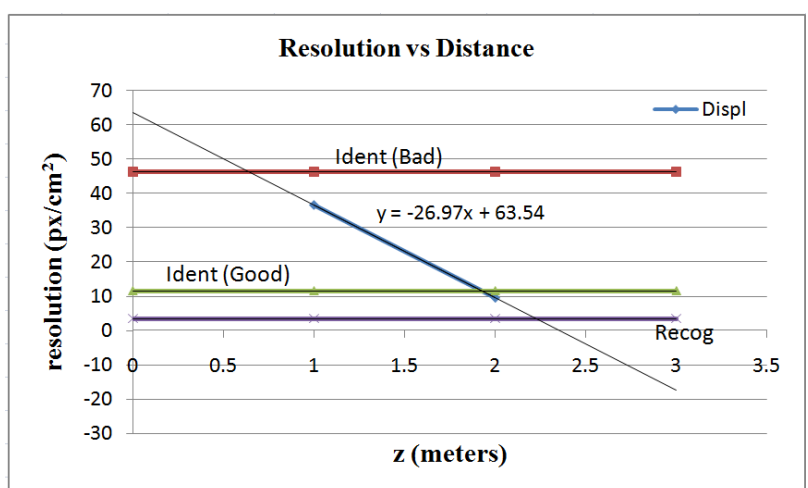

Fig. 3. A graph showing the effect of the resolution change as the distance between the camera and an object change on the detection of objects. 
In Fig. 3, three lines were drawn parallel to the distance $\mathrm{z}$ axis to present the three resolutions which were calculated in equations (5), (6) and (7). The three lines represented the resolution requirements for recognition, identification in good conditions and identification in bad conditions. A trend line was drawn for the two points which were calculated in equation (1) and (3). It crossed the five lines namely: resolution axis, distance $\mathrm{z}$-axis, identification in bad conditions, identification in good conditions and recognition lines. The equation of the trend line was a linear relation between the resolution axis and the distance $\mathrm{z}$-axis as shown in Fig. 3. From the equation, we found that in the distance range $z=0 m$ to $z=0.64 m$, the resolution ranged from 63.45 to $46.32 \mathrm{px} / \mathrm{cm}^{2}$ which was a suitable range for identification in bad conditions. In the distance range $z=0.64 m$ to $z=1.93 m$, the resolution ranged from 46.32 to $11.58 \mathrm{px} / \mathrm{cm}^{2}$ which was a suitable range for identification in good conditions. In the distance range $z=1.93 \mathrm{~m}$ to $z=2.23 \mathrm{~m}$, the resolution ranged from 11.58 to $3.47 \mathrm{px} / \mathrm{cm}^{2}$ which was a suitable range for human face recognition. These were the limits imposed on us through our system performance by the resolution of our cameras. Next, we discussed the experimental setup done to take stereo images and measured the accuracies of calculated distances from the detected objects to the camera used.

\section{Characteristics of Stereo CAMERA}

In this section, the functions used to calculate the disparity map from stereo images were illustrated. We started with the Gamma parameter which was responsible for lightening and darkening the images which were obtained by the cameras and changing their contrasts. Because the two cameras were not identical, so we had to modify the gamma value to make the two cameras had the same gray scale. Next, the WLS filter was used which was responsible for smoothing the edges of the images and changing the sharpness of the pictures to make the disparity more accurate. There were two types of WLS filter used [5] namely: the "wls_filter. setLambda", this filter was responsible for the smoothing ratio, and the "wls_filter. setSegmaColor", this filter was responsible for setting different colors. The two cameras were pointing away from each other. That was to say the position of a pixel in the right image was to the left of its corresponding position in the left image [5]. The distance B between the two axes of the two cameras was set to $0.1 \mathrm{~m}$. Next, openCV allowed us to calculate a disparity map from the two taken images. OpenCV used the Semi-global Block Matching algorithm inside the class StereoSGBM [5]. Dynamic programming was the method used to make the matching between the left and right cameras more error free. We used the command namely: "cv2.StereoSGBM_create(pararmeter1, parameter2, .....)" to adjust the two cameras to get the best disparity map that matches reality [9]. The values of the parameters used in the command were set through trial and error. We set the parameters minDisparity $=32$ and numDisparities $=128$. minDisparity referred to the difference in position of objects between right image and left image in pixels [9]. While, numDisparities referred to the maximum disparity minus minimum disparity [9] and was set to a large value just to be on the safe side. We set the parameter blockSize=3 which should typically had a value between 3 and 11 [9]. We needed to make this value minimum as it was the block size that needed to be searched for in the other image. Making it minimum made the search more accurate but took longer time while increasing the block size may had made it become impossible to found as too many objects were included. We set the parameters $\mathrm{P} 1=600$ and $\mathrm{P} 2=4800$. The values of $\mathrm{P} 1$ and P2 were penalties if disparity was not smooth [9]. We were acting on a continuous background in the images, so the penalty had a high value, to keep disparity very smooth. We were not having large differences in the distances between the objects and the cameras because the imaged place was an indoor area. We set the parameter of disparity maximum difference disp12MaxDiff=1 [9]. The disparity maximum difference between left and right images was set to one to disable the check for the maximum difference. If a maximum was set, then objects whose disparity exceeded this difference would be omitted which was not preferred. We didn't want to lose any information for the obtained images. We set the parameter uniquenessRatio $=150$ [9]. The uniquenessRatio stood for the percentage margin in cost function by which first match was defined and second best match was defined. Usually, it should be in the range 5 to 15 , but here the value was set to a very high one to retain the first match once found The difference in images was large due to the difference in the qualities of the two different cameras that was why this percentage was kept high. We set the parameters speckleWindowSize $=0$ and speckleRange $=1$ [9]. By setting off the speckleWindowSize, we disabled the speckle filtering as each individual image was good enough in this case and so as not to remove any details from the images. The speckleRange value was not valid since speckle filtering was disabled. We set the parameter preFilterCap=63 [9]. The preFilterCap referred to the difference in pixels values which were allowed, and it was set to a high value to allow wide variations in the pixels values. Also, we wanted to make sure that no information was truncated since the difference in the two images was large as they were taken using non-identical cameras. The two images obtained using the right and left cameras were shown in Fig. $4 \mathrm{a}$ and $4 \mathrm{~b}$ respectively.

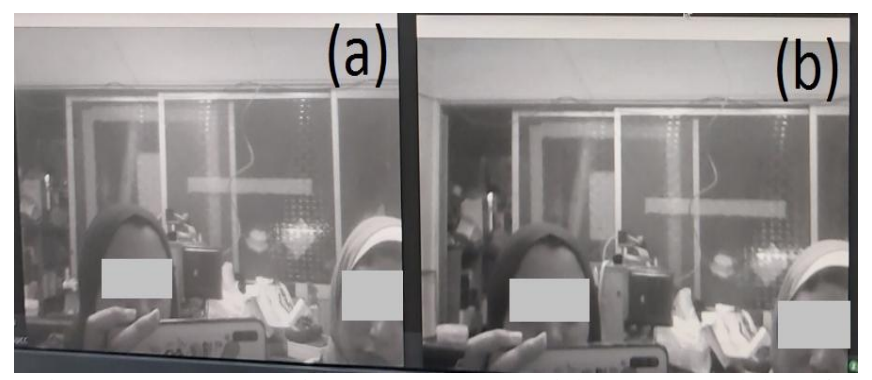

Fig. 4. Stereo camera using two cameras. (a) Right Image R and (b) Left image L.

As shown in Fig. 4, an image of the same indoor view was taken by the two cameras and the disparity map was calculated and shown in Fig. 5. 


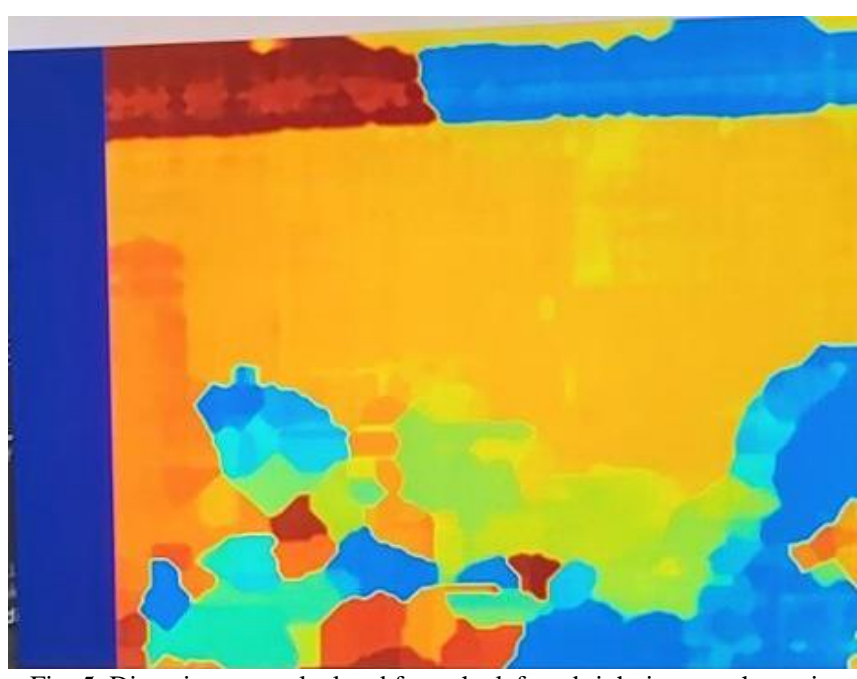

Fig. 5. Disparity map calculated from the left and right images shown in Fig. 4.

In Fig. 5, each color represented a different distance from the camera. The hot colors, such as red and yellow, represented larger distances between the camera and the objects while the cold colors, such as blue, represented shorter distances. Next, we studied the effect of disparity on the values of distances shown on the disparity map.

\section{NUMERICAL RESULTS}

In this section, we discussed the data which we collected using our stereo cameras. The theoretical equations which describe the relations between the parameters of the produced images were illustrated.

\section{A. Distance Detection versus Disparity}

In this subsection, we use the stereo cameras to take images and study the accuracy of finding the position of detected objects. Theoretically, the disparity $\left(x_{L}-x_{R}\right)$ is inversely proportional with the distance $z$ between an object and the camera. The equation governing the relation between disparity and distance was [6]:

$$
z=B x_{0} /\left(2 \tan \left(\frac{\phi_{0}}{2}\right)\left(x_{L}-x_{R}\right)\right)
$$

where $x_{L}$ was the position of object in left image (in pixels) while $x_{R}$ was the position of the same object in the right image (in pixels). The constant of proportionality in equation (8) was a function of: the distance between the two axes of the two cameras which was called baseline $B$, the horizontal number of pixels scanned by one camera $x_{0}$, and the angle of view scanned by one camera which was $B$. In Table I, we recorded the values for the distance parameter $\mathrm{z}$ against different values of disparity $\left(x_{L}-x_{R}\right)$.

TABLE I: DisPaRity VALUES AS SHOWN IN THE DiSPARITY MAP (FIG. 5) AND THE CORRESPONDING Z VALUES (THEORETICAL, DisPlaYED (FIG. 5)

\begin{tabular}{cccc}
\multicolumn{4}{c}{ AND ACTUAL $)$} \\
\hline Disparity $(\mathrm{px})$ & $\mathrm{z}$ (theor) & $\mathrm{z}($ displ $)$ & $\mathrm{z}($ actual $)$ \\
\hline 51 & 1.18 & 1.06 & 1.08 \\
43 & 1.40 & 1.25 & 1.3 \\
40 & 1.50 & 1.37 & 1.43 \\
35 & 1.72 & 1.53 & 1.56 \\
\hline
\end{tabular}

In Table I, the first column stood for the disparity values as displayed on the disparity map which was shown in Fig. 5. The second column represented the calculated theoretical values of the distance $\mathrm{z}$ for each value of disparity mentioned in the first column using equation (8). In equation (8), we used the following values: $\phi_{o}=55^{\circ}, B=0.1 \mathrm{~m}$ and $x_{o}=640 p x$. The displayed values of distance $\mathrm{z}$ from the disparity map shown in Fig. 5 were tabulated in the third column. In the fourth column, the actual values of the distance $\mathrm{z}$ were measured by a $30 \mathrm{~cm}$ ruler and recorded. The values of disparity were inversely proportional with the values tabulated in the three columns. Next, we studied the variation in the error of the distance displayed on the disparity map which was shown in Fig. 5.

\section{B. Error in Distance versus Distance}

In this subsection, we compared the error in measuring the distance $z$ of each object. Theoretically, the error in distance $\Delta z$ was directly proportional with the square of the distance $z$ between the object and the camera. The equation governing the relation between the distance $\mathrm{z}$ and the error in it was [6]:

$$
\Delta z=\left(z^{2} / B\right) \tan \left(\phi_{0} / x_{0}\right)
$$

where the variables $\phi_{o}, B$ and $x_{o}$ stood for the same parameters mentioned in equation (8). In Table II, we recorded two values for the error in the distance parameter $\Delta \mathrm{z}$ against different values of the distance $z$.

TABLE II: ZVALUES AS SHOWN IN THE DISPARITY MAP (FIG. 5) AND THE

\begin{tabular}{|c|c|c|}
\hline $\mathrm{z}($ displ $)$ & $\Delta \mathrm{z}$ (theo) & $\Delta \mathrm{z}($ dis-act $)$ \\
\hline 1.06 & 0.02 & 0.02 \\
\hline 1.25 & 0.02 & 0.05 \\
\hline 1.37 & 0.03 & 0.06 \\
\hline 1.53 & 0.04 & 0.03 \\
\hline
\end{tabular}
CorResPonding ERror IN THE Z VALUES (TheORETICAL $\Delta z$ AND $\Delta z$

In Table II, the first column stood for the distance $\mathrm{z}$ values as displayed on the disparity map which was shown in Fig. 5. The second column was the calculated theoretical values of the error in the distance $\Delta \mathrm{z}$ for each value of $\mathrm{z}$ mentioned in the first column using (9). In equation (9), we used the same values for: $\phi_{o}, B$ and $x_{o}$ as those used in (8). The difference $\Delta \mathrm{z}$ (error) between the displayed values of the distance $\mathrm{z}$ and the actual values of the distance $\mathrm{z}$ (both shown in Table I column 3 and 4) was tabulated in the third column. The theoretical error $\Delta \mathrm{z}$ was almost half the calculated error $\Delta \mathrm{z}$ which was shown in the third column in Table II. Next, we discussed the results recorded in this paper and drew our conclusion.

\section{DISCUSSION AND CONCLUSION}

In this section, the results obtained throughout the paper was discussed and plotted when applicable. The resolution of each camera and the rate of change of resolution with distance of objects put limits on object recognition. Here we have used a simple manual method of perpendicular rulers to find the range of distances from the camera that was suitable for 
recognition, identification in good conditions and identification in bad conditions. Then, we discussed the dependence of the distance $z$ (from object to camera) on the disparity measure. The results tabulated in Table I were shown in Fig. 6.

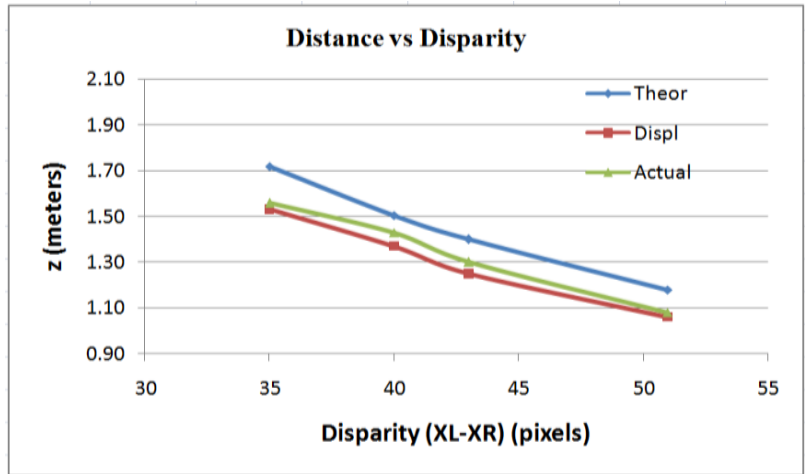

Fig. 6. A graph showing the inverse relation between Distance and disparity.

In Fig.6, the theoretical values of the distance $\mathrm{z}$ calculated using (8), the displayed distance $\mathrm{z}$ in the disparity map shown in Fig. 5 and the actual distance between the cameras and the objects imaged were shown. Displayed and actual distances were almost identical, but the theoretical values were larger than both of them. The error can be due to optical errors such as barrel, pincushion distortion, aberration, etc. In addition, the method of measuring the actual distances $\mathrm{z}$ was done by using a $30 \mathrm{~cm}$ ruler which was suitable for rough measurements but not accurate enough for comparison with the theoretical results. Next, the logarithmic to the base 10 of the results tabulated in Table II was shown in the graph of Fig. 7.

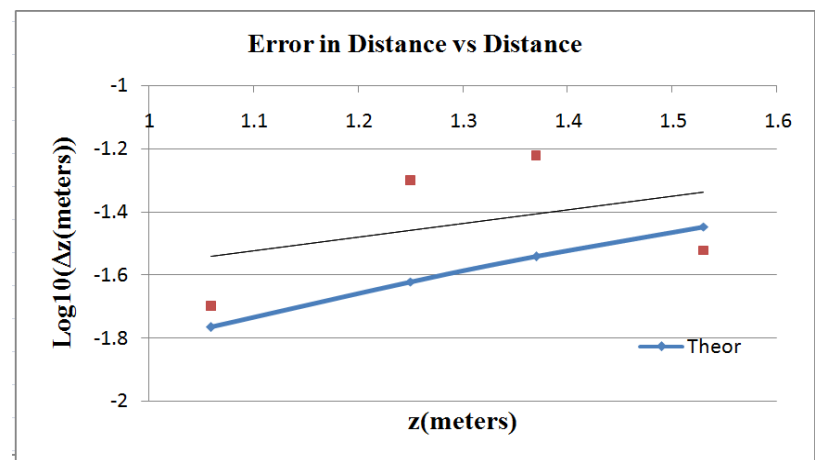

Fig. 7. A graph showing the direct relation between error in distance and distance shown on disparity map.

In Fig. 7 the theoretical calculation of the error in distance using (9) and the difference between displayed distance on the disparity map and the actual distance were plotted. The trend line representing the error in distance between displayed and actual had higher values than the line representing the theoretical error. That was because the error was measured using a $30 \mathrm{~cm}$ ruler which would be a source of error in itself if the differences between actual and displayed was in millimeters and centimeters as was the case here. In addition, better accuracy could be obtained with wider distance between the two axes of the two cameras [10]. Here the distance between the two axes of the cameras $B$ was limited by the size of the holder on which the two cameras was held. If B was set to a higher value, the error in distance could be reduced.

Using two non-identical cameras to obtain stereo images was possible. But a series of trial and error experimental work had to be carried out to reach the best possible accuracy. If used in the outside doors' applications, the error would be huge since in this case the highest number of pixels per unit area would be needed especially if human face identification was required.

\section{CONFLICT OF INTEREST}

Authors declare that they do not have any conflict of interest.

\section{REFERENCES}

[1] Research Note on Impacts of COVID-19 Pandemic on Egypt's Economy; March 2020; American Chamber of Commerce in Egypt (AmCham Egypt).

[2] Christodoulou, Lakis. 3D Stereo Vision Camera-sensors, Advancements, and Technologies, 2013.

[3] Object-Centric Stereo Matching for 3D Object Detection; Alex D. Pon and Jason $\mathrm{Ku}$ and Chengyao $\mathrm{Li}$ and Steven L. Waslander; arXiv:1909.07566v2 [cs.CV1] 10 Mar 2020.

[4] https://www.jetbrains.com/pycharm/download/\#section=windows.

[5] https://opencv-python-

tutroals.readthedocs.io/en/latest/py_tutorials/py_calib3d/py_depthmap /py_depthmap.html.

[6] Distance measuring based on stereoscopic pictures, Jernej Mrovlje and Damir Vrancic, 9th International PhD Workshop on Systems and Control: Young Generation Viewpoint 1-3. October 2008, Izola, Slovenia.

[7] DU LL, Wang LM, Zhuang Z. [Measurement and analysis of human head-face dimensions1]. Zhonghua Lao Dong Wei Sheng Zhi Ye Bing Za Zhi. 2008 May;26(5) 266-270. PMID: 18727867.

[8] How Many Pixels Do We Need to See Things? Yang Cai, P.M.A. Sloot et al. (Eds.): ICCS 2003, LNCS 2659, pp. 1064-1073, 2003. SpringerVerlag Berlin Heidelberg 2003.

[9] https://docs.opencv.org/3.4/d2/d85/classcv_1_1StereoSGBM.html.

[10] On the Importance of Stereo for Accurate Depth Estimation: An Efficient Semi-Supervised Deep Neural Network Approach; Nikolai Smolyanskiy Alexey Kamenev Stan Birchfield; arXiv:1803.09719v4 [cs.CV1] 8 Jul 2020.

[11] Johnston KJ, Hammond G, Meyers DJ, Joynt Maddox KE. Association of race and ethnicity and medicare program type with ambulatory care access and quality measures. JAMA. 2021 Aug 17;326(7):628-636. doi: 10.1001/jama.2021.10413.

[12] Naderi SH, Bestwick JP, Wald DS. Adherence to drugs that prevent cardiovascular disease: meta-analysis on 376,162 patients. Am J Med 2012; 125(9): 882-887.e1. 